\title{
Analog Beam Selection Schemes of DFT-based Hybrid Beamforming Multiuser Systems
}

\author{
Yu Han*, Shi Jin*, Jun Zhang ${ }^{\dagger}$, and Jiayi Zhang ${ }^{\ddagger}$, and Kai-Kit Wong ${ }^{\S}$ \\ *National Mobile Communications Research Laboratory, Southeast University, Nanjing, China \\ ${ }^{\dagger}$ Nanjing University of Posts and Telecommunications, Nanjing, China \\ ${ }^{\ddagger}$ School of Electronic and Information Engineering, Beijing Jiaotong University, Beijing, China \\ $\S$ Department of Electronic and Electrical Engineering, University College London, United Kingdom
}

\begin{abstract}
This paper studies analog beam selection schemes of discrete Fourier transform (DFT) based hybrid beamforming systems. We first derive approximations of the achievable rates when maximum-ratio combining (MRC) receiver and maximumratio transmitting (MRT) precoder are used in the uplink and downlink, respectively. It is shown that the achievable rate of the hybrid beamforming system is improved with the increase of the number of radio frequency chains. Also, it is found that the orthogonality condition among the line-of-sight (LoS) paths from different users directly determines the interference cancellation capability of the MRC receiver or the MRT precoder Based on our analytical results, we propose two novel DFT beam selection schemes, referred to as exhausted searching and per-user selection. Numerical results show that the first scheme achieves higher rate while the second one is a simple suboptimal strategy with low complexity, which is practically more attractive.
\end{abstract}

\section{INTRODUCTION}

Massive multiple-input multiple-output (MIMO) is a key technology in the fifth-generation mobile communication systems, e.g., [1], [2]. By deploying a large-scale antenna array at the base station (BS), massive MIMO can produce extremely high spatial resolution and increase multiuser spatial division dimensions [3], [4]. However, the cost of fully digital massive MIMO system is expensive due to the huge number of expensive radio frequency (RF) chains. Thus, a hybrid beamforming structure has been proposed, which utilizes a small number of RF chains to control the large scale antenna array [5], [6]. Hybrid beamforming structure has been widely accepted as a low-cost solution of massive MIMO practical employments.

In the context of multiuser MIMO systems, lots of work have been conducted. In [7], [8], the authors studied the performance of a multiuser spatial multiplexing system when the zero-forcing receiver was used at the BS. Then [8] compared the uplink achievable rate in Rayleigh and Ricean fading conditions, while [9] formulated the boundaries of the achievable rate of a multiuser massive MIMO system and compared the performance for various precoders and receivers. The results were limited to fully digital systems and cannot be used for hybrid beamforming systems. In [10], a low-complexity hybrid beamforming solution was proposed based on a low-dimensional baseband zero-forcing precoding. However, only the Rayleigh fading was considered, which is not the key propagation scenario suitable for hybrid structures.
In this paper, we focus on the investigation of discrete Fourier transform (DFT) based beam selection schemes of the multiuser hybrid beamforming system. Ricean fading condition is considered due to its great importance in concurrent and future applications such as millimeter-wave and machine-type communications. Maximum-ratio combining (MRC) receiver and maximum-ratio transmitting (MRT) precoder are adopted. We assume that perfect channel state information (CSI) is known at the BS. First, an approximation of the uplink achievable rate is derived. We find that the orthogonality condition among the line-of-sight (LoS) paths from different users largely influences the performance of MRC receivers. When the Ricean $K$-factor is enhanced, the achievable rate increases and converges. Then, for the downlink, we adopt long-term and short-term normalization methods and formulate their corresponding achievable rate approximations. Based on the analytical results, we propose two beam selection schemes. The first one is called the exhausted searching scheme, which selects the optimum beam combination that maximizes the achievable rate. The second one is the peruser selection scheme, which is simple but efficient. Numerical results demonstrate the high rate performance of the proposed beam selection schemes. It is also shown that the per-user selection scheme performs nearly the same as the exhausted searching solution with inappreciable difference.

\section{System Model}

We consider a massive MIMO system operating in timedivision-duplex (TDD) mode. The BS is equipped with $M$ antennas and communicates with $N_{u}$ single-antenna users simultaneously. The uplink channel can be written as

$$
\mathbf{G}=\mathbf{H D}^{\frac{1}{2}},
$$

where $\mathbf{D}=\operatorname{diag}\left\{\beta_{1}, \beta_{2}, \ldots, \beta_{N_{u}}\right\}$ is the large-scale fading factor matrix which reflects the energy of each user channel, and $\mathbf{H} \in \mathbb{C}^{M \times N_{u}}$ is the fast fading factor matrix which describes the propagation characteristic of the channel. Here, the following Ricean channel model is considered:

$$
\mathbf{H}=\overline{\mathbf{H}}\left[\boldsymbol{\Omega}(\boldsymbol{\Omega}+\mathbf{I})^{-1}\right]^{\frac{1}{2}}+\mathbf{H}_{w}\left[(\boldsymbol{\Omega}+\mathbf{I})^{-1}\right]^{\frac{1}{2}},
$$




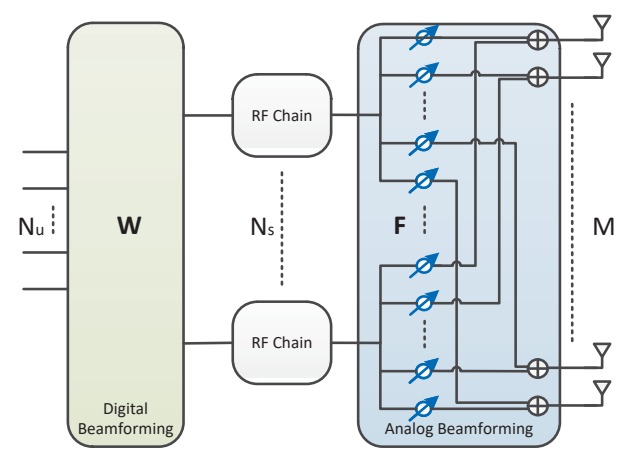

Fig. 1. Full-connection hybrid beamforming structure at the BS. There are totally $M \times N_{s}$ phase shifters.

where $\boldsymbol{\Omega}=\operatorname{diag}\left\{K_{1}, K_{2}, \ldots, K_{N_{u}}\right\}$ is the Ricean $\mathrm{K}$-factor matrix, $\overline{\mathbf{H}} \in \mathbb{C}^{M \times N_{u}}$ is the deterministic LoS component, $\mathbf{H}_{w} \in \mathbb{C}^{M \times N_{u}}$ is the random component with i.i.d. elements, and each element is a complex Gaussian random variable with zero mean and unit variance. Due to the reciprocity of TDD system, the multiuser downlink channel is $\mathbf{G}^{T}$.

The BS adopts a hybrid beamforming structure. There are totally $N_{s}\left(N_{u} \leq N_{s} \leq M\right)$ RF chains. Each RF chain connects with all the antenna elements. Each connection between an RF chain and an antenna is controlled by a phase shifter. The full-connection hybrid structure is shown in Fig. 1.

In the uplink, MRC is adopted at the BS. Assume that the uplink transmit power is averaged out and all the users have equal transmit power. The uplink signal can be modeled as

$$
\mathbf{y}=\sqrt{P_{a v g}} \mathbf{G}_{e q}^{H} \mathbf{F} \mathbf{G s}+\mathbf{G}_{e q}^{H} \mathbf{F n},
$$

where $P_{a v g}$ is the average transmit power of each user, $\mathbf{G}_{e q}=$ FG is the effective channel seen from the air interface and $\mathbf{G}_{e q}^{H}$ represents the MRC receiver, $\mathbf{F} \in \mathbb{C}^{N_{s} \times M}$ is the analog beamforming matrix, $\mathbf{s} \in \mathbb{C}^{N_{u} \times 1}$ is the transmit signal vector satisfying $\mathbb{E}\left\{\mathbf{s s}^{H}\right\}=\mathbf{I}_{N_{u}}$, and $\mathbf{n} \in \mathbb{C}^{M \times 1}$ is the complex noise vector with each element having zero mean and unit variance.

Also, the MRT precoder is employed in the downlink. The received signal vector at the user side is expressed as

$$
\mathbf{y}=\sqrt{P} \mathbf{G}^{T} \mathbf{F}^{T} \mathbf{G}_{e q}^{*} \mathbf{\Lambda} \mathbf{x}+\mathbf{n},
$$

where $P$ is the total transmit power at the $\mathrm{BS}, \mathbf{G}_{e q}^{*}$ represents the MRT precoder, $\boldsymbol{\Lambda}=\operatorname{diag}\left\{\rho_{1}, \rho_{2}, \ldots, \rho_{N_{u}}\right\}$ is the power normalization matrix, $\mathbf{x} \in \mathbb{C}^{N_{u} \times 1}$ is the transmit signal vector satisfying $\mathbb{E}\left\{\mathbf{x x}^{H}\right\}=\mathbf{I}_{N_{u}}$, and $\mathbf{n} \in \mathbb{C}^{N_{u} \times 1}$ is the complex noise vector with each element having unit variance.

For convenience, we introduce the DFT codebook which divides the whole space into $M$ orthogonal beams. Denote the $M$ dimensional DFT matrix as

$$
\mathbf{U}=\frac{1}{\sqrt{M}}\left[\begin{array}{cccc}
1 & 1 & \cdots & 1 \\
1 & e^{j 2 \pi \frac{1}{M}} & \cdots & e^{j 2 \pi \frac{M-1}{M}} \\
\vdots & \vdots & & \vdots \\
1 & e^{j 2 \pi \frac{M-1}{M}} & \cdots & e^{j 2 \pi \frac{(M-1)^{2}}{M}}
\end{array}\right]
$$

The analog beamforming matrix is constructed with $N_{s}$ rows or columns of $\mathbf{U}$, that is, $\mathbf{F}=\boldsymbol{\Psi}^{\mathrm{T}} \mathbf{U}$, where $\boldsymbol{\Psi}=$ $\left[\mathbf{e}_{i_{1}}, \mathbf{e}_{i_{2}}, \ldots, \mathbf{e}_{i_{N_{s}}}\right]$ and $\mathbf{e}_{j} \in \mathbb{Z}^{M \times 1}$ is a vector with $j$ th element equals to 1 and others equal to 0 .

\section{Achievable Rate AnAlysis}

In this section, we focus on the achievable rate analysis of this DFT-based hybrid beamforming multiuser system with fixed analog beamforming matrices $\mathbf{F}$ when MRC receiver and MRT precoder are employed in uplink and downlink, respectively.

\section{A. Uplink}

According to (3), the uplink received signal y contains both the original transmit signal and the noise. It should be noted that the noise is also beamformed. Since $\mathbf{F}$ is the abstraction from DFT matrix $\mathbf{U}$ and $\mathbf{n}$ is a multivariable Gaussian random vector, the colored noise $\mathbf{F n}$ can be seen as a new $N_{s^{-}}$ dimensional Gaussian vector $\hat{\mathbf{n}}$ with each element satisfying a distribution of $\mathcal{N}(0,1)$. Therefore, the data stream to be detected for user $k$ can be decomposed as

$$
y_{k}=\sqrt{P_{a v g}} \mathbf{g}_{e q, k}^{H} \mathbf{g}_{e q, k} s_{k}+\sum_{j \neq k} \sqrt{P_{a v g}} \mathbf{g}_{e q, k}^{H} \mathbf{g}_{e q, j} s_{j}+\mathbf{g}_{e q, k}^{H} \hat{\mathbf{n}}_{k} .
$$

In this condition, the system achievable rate becomes

$$
R^{\mathrm{MRC}}=\sum_{k=1}^{N_{u}} \mathbb{E}\left\{\log _{2}\left(1+\gamma_{k}\right)\right\}
$$

where

$$
\gamma_{k}=\frac{P_{a v g}\left\|\mathbf{g}_{e q, k}\right\|^{4}}{\sum_{j \neq k} P_{a v g}\left|\mathbf{g}_{e q, k}^{H} \mathbf{g}_{e q, j}\right|^{2}+\left\|\mathbf{g}_{e q, k}\right\|^{2}}
$$

is the signal-to-interference plus noise ratio (SINR), and $\mathbf{g}_{e q, k}$ is the $k$ th column vector of $\mathbf{G}_{e q}$. For better understanding of the rate performance, we derive the following theorem.

Theorem 1: When adopting the MRC receiver in the DFTbased multiuser hybrid beamforming system, the uplink achievable rate can be approximated as

$$
R_{\mathrm{App}}^{\mathrm{MRC}}=\sum_{k=1}^{N_{u}} \log _{2}\left(1+\frac{\frac{P_{a v g} \beta_{k}}{K_{k}+1} \chi_{1}^{(k)}}{\sum_{j \neq k} \frac{P_{a v g} \beta_{j}}{K_{j}+1} \chi_{2, j}^{(k)}+\chi_{3}^{(k)}}\right)
$$

where

$$
\begin{aligned}
& \chi_{3}^{(k)} \triangleq K_{k}\left\|\mathbf{F} \overline{\mathbf{h}}_{k}\right\|^{2}+N_{s}, \\
& \chi_{1}^{(k)} \triangleq \chi_{3}^{(k) 2}+2 \chi_{3}^{(k)}-N_{s},
\end{aligned}
$$

and

$$
\chi_{2, j}^{(k)} \triangleq K_{k} K_{j}\left|\overline{\mathbf{h}}_{j}^{H} \mathbf{F}^{H} \cdot \mathbf{F} \overline{\mathbf{h}}_{k}\right|^{2}+\chi_{3}^{(j)}+\chi_{3}^{(k)}-N_{s} .
$$


Proof: First, let us refer to Lemma 1 of [11] and calculate the achievable rate of user $k$ as

$$
\begin{aligned}
& R_{k}^{\mathrm{MRC}} \approx \\
& \log _{2}\left(1+\frac{P_{a v g} \mathbb{E}\left\{\left\|\mathbf{g}_{e q, k}\right\|^{4}\right\}}{\sum_{j \neq k} P_{a v g} \mathbb{E}\left\{\left|\mathbf{g}_{e q, k}^{H} \mathbf{g}_{e q, j}\right|^{2}\right\}+\mathbb{E}\left\{\left\|\mathbf{g}_{e q, k}\right\|^{2}\right\}}\right) .
\end{aligned}
$$

According to the definition of $\mathbf{G}_{e q}$, we can further write

$$
\mathbf{g}_{e q, k}=\mathbf{F g}_{k}=\sqrt{\frac{\beta_{k}}{K_{k}+1}} \mathbf{F h}_{w, k}+\sqrt{\frac{K_{k} \beta_{k}}{K_{k}+1}} \mathbf{F} \overline{\mathbf{h}}_{k},
$$

where $\mathbf{h}_{w, k}$ and $\overline{\mathbf{h}}_{k}$ are the $k$ th column vector of $\mathbf{H}_{w}$ and $\overline{\mathbf{H}}$, respectively. Recalling the feature of the DFT transformation of a Gaussian vector, we know that $\mathbf{F h}_{w, k}$ is still an $N_{s}$ dimensional Gaussian vector. Hence, after some derivations, we get the expression of the third expectation item in (13) as

$$
\begin{aligned}
\mathbb{E}\left\{\left\|\mathbf{g}_{e q, k}\right\|^{2}\right\} & =\frac{\beta_{k}}{K_{k}+1}\left(N_{s}+K_{k}\left\|\mathbf{F} \overline{\mathbf{h}}_{k}\right\|^{2}\right) \\
& =\frac{\beta_{k}}{K_{k}+1} \chi_{3}^{(k)},
\end{aligned}
$$

and the first and the second expectation items can be found in (16) and (17) respectively at the bottom of this page. Applying (15)-(17) into (13), we can get the desired result.

To obtain more insights on Theorem 1, we consider the following special cases.

Case 1: When $K_{k}=0$ for $k=1, \ldots, N_{u}$, Ricean fading reduces to Rayleigh fading, and (9) is rewritten as

$$
R_{\mathrm{App}}^{\mathrm{MRC}}=\sum_{k=1}^{N_{u}} \log _{2}\left(1+\frac{P_{a v g} \beta_{k}\left(N_{s}+1\right)}{\sum_{j \neq k} P_{a v g} \beta_{j}+1}\right) .
$$

We can see that the achievable rate of the hybrid beamforming system grows with the increase of the number of RF chains. Moreover, when $N_{s}=M$ and $\beta_{1}=\beta_{2}=\cdots=\beta_{N_{u}}=1$, the derived expression is exactly in accordance with [11].

Case 2: In case that $K_{k}, K_{j} \rightarrow \infty$, the achievable rate approaches

$$
R_{\mathrm{App}}^{\mathrm{MRC}}=\sum_{k=1}^{N_{u}} \log _{2}\left(1+\frac{P_{a v g} \beta_{k}\left\|\mathbf{F} \overline{\mathbf{h}}_{k}\right\|^{4}}{\sum_{j \neq k} P_{a v g} \beta_{j}\left|\overline{\mathbf{h}}_{j}^{H} \mathbf{F}^{H} \mathbf{F} \overline{\mathbf{h}}_{k}\right|^{2}+\left\|\mathbf{F} \overline{\mathbf{h}}_{k}\right\|^{2}}\right) .
$$

When the effective LoS components further hold orthogonality, i.e., $\overline{\mathbf{h}}_{j}^{H} \mathbf{F}^{H} \mathbf{F} \overline{\mathbf{h}}_{k}=0$ for $j \neq k$, (19) can be reduced to

$$
R_{\mathrm{App}}^{\mathrm{MRC}} \rightarrow \sum_{k=1}^{N_{u}} \log _{2}\left(1+P_{a v g} \beta_{k}\left\|\mathbf{F} \overline{\mathbf{h}}_{k}\right\|^{2}\right) .
$$

Case 3: In case that $K_{k}, K_{j} \rightarrow \infty$, when the users stay together and share the same LOS component, that is, $\overline{\mathbf{h}}_{k}=\overline{\mathbf{h}}_{j}$ for $j \neq k$, then (19) is reduced to

$$
R_{\mathrm{App}}^{\mathrm{MRC}}=\sum_{k=1}^{N_{u}} \log _{2}\left(1+\frac{P_{a v g} \beta_{k}\left\|\mathbf{F} \overline{\mathbf{h}}_{k}\right\|^{2}}{\sum_{j \neq k} P_{a v g} \beta_{j}\left\|\mathbf{F} \overline{\mathbf{h}}_{k}\right\|^{2}+1}\right) .
$$

Considering (20) and (21), we can find that in Ricean fading scenario, the orthogonality condition among LoS paths from different users directly determines the interference cancellation capability of the MRC receiver. If the orthogonality holds among the analog beamformed LoS paths, no interference exists and the achievable rate can be enhanced by increasing $N_{s}$. Furthermore, if the analog beamformed LoS paths are highly correlated, the MRC receiver cannot work well in interference cancellation even by increasing $N_{s}$.

\section{B. Downlink}

In the downlink, considering the power normalization, the system achievable rate is calculated as

$$
R^{\mathrm{MRT}}=\sum_{k=1}^{N_{u}} \mathbb{E}\left\{\log _{2}\left(1+\frac{P \rho_{k}^{2}\left\|\mathbf{g}_{e q, k}\right\|^{4}}{\sum_{j \neq k} P \rho_{j}^{2}\left|\mathbf{g}_{e q, k}^{H} \mathbf{g}_{e q, j}\right|^{2}+1}\right)\right\} .
$$

We introduce two kinds of power normalization method. The first method is called long-term normalization and the power allocation factors are designed as

$$
\rho=\rho_{1}=\rho_{2}=\cdots=\rho_{N_{u}}=\frac{1}{\sqrt{\mathbb{E}\left\{\left\|\mathbf{G}_{e q}\right\|_{F}^{2}\right\}}} .
$$

In this method, long-term CSI is utilized to adjust $\Lambda$. To evaluate the performance of long-term normalization, we derive the following theorem.

Theorem 2: When adopting MRT precoding and long-term normalization method, the downlink achievable rate approxi-

$$
\begin{aligned}
& \mathbb{E}\left\{\left\|\mathbf{g}_{e q, k}\right\|^{4}\right\}=\frac{\beta_{k}^{2}}{\left(K_{k}+1\right)^{2}}\left[K_{k}^{2}\left\|\mathbf{F} \overline{\mathbf{h}}_{k}\right\|^{4}+2 K_{k}\left(N_{s}+1\right)\left\|\mathbf{F} \overline{\mathbf{h}}_{k}\right\|^{2}+N_{s}\left(N_{s}+1\right)\right]=\frac{\beta_{k}^{2}}{\left(K_{k}+1\right)^{2}}\left(\chi_{3}^{(k) 2}+2 \chi_{3}^{(k)}-N_{s}\right. \\
& \mathbb{E}\left\{\left|\mathbf{g}_{e q, k}^{H} \mathbf{g}_{e q, j}\right|^{2}\right\}=\frac{\beta_{k} \beta_{j}}{\left(K_{k}+1\right)\left(K_{j}+1\right)}\left(K_{k} K_{j}\left|\overline{\mathbf{h}}_{j}^{H} \mathbf{F}^{H} \cdot \mathbf{F} \overline{\mathbf{h}}_{k}\right|^{2}+K_{k}\left\|\mathbf{F} \overline{\mathbf{h}}_{k}\right\|^{2}+K_{j}\left\|\mathbf{F} \overline{\mathbf{h}}_{j}\right\|^{2}+N_{s}\right) \\
&=\frac{\beta_{k} \beta_{j}}{\left(K_{k}+1\right)\left(K_{j}+1\right)}\left(K_{k} K_{j}\left|\overline{\mathbf{h}}_{j}^{H} \mathbf{F}^{H} \cdot \mathbf{F} \overline{\mathbf{h}}_{k}\right|^{2}+\chi_{3}^{(j)}+\chi_{3}^{(k)}-N_{s}\right) .
\end{aligned}
$$


mates to

$$
\begin{aligned}
& R_{\mathrm{App}}^{\mathrm{MRT} 1}= \\
& \sum_{k=1}^{N_{u}} \log _{2}\left(1+\frac{\frac{P \beta_{k}^{2}}{\left(K_{k}+1\right)^{2}} \chi_{1}^{(k)}}{\sum_{j \neq k} \frac{P \beta_{k} \beta_{j}}{\left(K_{k}+1\right)\left(K_{j}+1\right)} \chi_{2, j}^{(k)}+\sum_{i=1}^{N_{u}} \frac{\beta_{i}}{K_{i}+1} \chi_{3}^{(i)}}\right),
\end{aligned}
$$

where $\chi_{1}^{(k)}, \chi_{2}^{(k)}$ and $\chi_{3}^{(k)}$ are defined in (10)-(12).

Proof: According to Lemma 1 of [11], (22) can be approximated by

$$
R^{\mathrm{MRT} 1} \approx \sum_{k=1}^{N_{u}} \log _{2}\left(1+\frac{P \rho_{k}^{2} \mathbb{E}\left\{\left\|\mathbf{g}_{e q, k}\right\|^{4}\right\}}{\sum_{j \neq k} P \rho_{j}^{2} \mathbb{E}\left\{\left|\mathbf{g}_{e q, k}^{H} \mathbf{g}_{e q, j}\right|^{2}\right\}+1}\right),
$$

where the expressions of the two expectation items can be found in (16) and (17). When it comes to $\rho_{k}^{2}$, we can refer to the definition of long-term normalization in (23) and obtain

$$
\begin{aligned}
& \mathbb{E}\left\{\left\|\mathbf{G}_{e q}\right\|_{F}^{2}\right\}=\mathbb{E}\left\{\sum_{i=1}^{N_{u}}\left\|\mathbf{g}_{e q, i}\right\|^{2}\right\} \\
= & \sum_{i=1}^{N_{u}} \frac{\beta_{i}}{K_{i}+1}\left(N_{s}+K_{i}\left\|\mathbf{F} \overline{\mathbf{h}}_{i}\right\|^{2}\right)=\sum_{i=1}^{N_{u}} \frac{\beta_{i}}{K_{i}+1} \chi_{3}^{(i)} .
\end{aligned}
$$

Applying (16), (17) and (26) into (25), we can obtain (24).

From the definition of long-term normalization for MRT precoding we can find that the matrix $\boldsymbol{\Lambda}$ remains unchanged for the coherence time of channels between BS and users. Hence, the long-term normalization cannot trace the smallscale fading of the channel. Here, we further use the second method which is called short-term normalization, where $\boldsymbol{\Lambda}$ is derived according to instantaneous CSI. In this method,

$$
\rho_{k}=\frac{1}{\sqrt{N_{u}}\left\|\mathbf{g}_{e q, k}\right\|},
$$

which means that each user's data stream is allocated with equal transmit power. Moreover, it requires more frequently CSI calculation and exchange. Similarly, we get the following theorem to help analyze the short-term normalization.

Theorem 3: When adopting MRT precoding and short-term normalization method, the downlink achievable rate can be approximated as

$$
R_{\mathrm{App}}^{\mathrm{MRT} 2}=\sum_{k=1}^{N_{u}} \log _{2}\left(1+\frac{\frac{P \beta_{k}}{N_{u}\left(K_{k}+1\right)} \chi_{3}^{(k)}}{\sum_{j \neq k} \frac{P \beta_{k}}{N_{u}\left(K_{k}+1\right)} \frac{\chi_{2, j}^{(k)}}{\chi_{3}^{(j)}}+1}\right) .
$$

Proof: According to the definition of short-term normalization, the achievable rate satisfies

$$
R^{\mathrm{MRT} 2}=\sum_{k=1}^{N_{u}} \mathbb{E}\left\{\log _{2}\left(1+\frac{\frac{P}{N_{u}}\left\|\mathbf{g}_{e q, k}\right\|^{2}}{\sum_{j \neq k} \frac{P\left|\mathbf{g}_{e q, k}^{H} \mathbf{g}_{e q, j}\right|^{2}}{N_{u}\left\|\mathbf{g}_{e q, j}\right\|^{2}}+1}\right)\right\} .
$$

Recalling Lemma 1 of [11], (29) can be approximated by

$$
R^{\mathrm{MRT} 2} \approx \sum_{k=1}^{N_{u}} \log _{2}\left(1+\frac{\frac{P}{N_{u}} \mathbb{E}\left\{\left\|\mathbf{g}_{e q, k}\right\|^{2}\right\}}{\sum_{j \neq k} \frac{P \mathbb{E}\left\{\left|\mathbf{g}_{e q, k}^{H} \mathbf{g}_{e q, j}\right|^{2}\right\}}{N_{u} \mathbb{E}\left\{\left\|\mathbf{g}_{e q, j}\right\|^{2}\right\}}+1}\right) .
$$

Utilizing the results in (15) and (17), we can obtain (28).

To compare the performance of long-term and short-term normalization, we consider the following special cases.

Case 1: When reduced to Rayleigh fading condition, (24) and (28) are rewritten as

$$
R_{\mathrm{App}}^{\mathrm{MRT} 1}=\sum_{k=1}^{N_{u}} \log _{2}\left(1+\frac{P \beta_{k}^{2}\left(N_{s}+1\right)}{\sum_{j \neq k} P \beta_{k} \beta_{j}+\sum_{k=1}^{N_{u}} \beta_{i}}\right)
$$

and

$$
R_{\mathrm{App}}^{\mathrm{MRT} 2}=\sum_{k=1}^{N_{u}} \log _{2}\left(1+\frac{P \beta_{k} N_{s}}{P \beta_{k}\left(N_{u}-1\right)+N_{u}}\right) .
$$

Case 2: In the case that $K_{k}, K_{j} \rightarrow \infty$, if orthogonality holds among the effective LOS components of different users, (24) and (28) are reduced to, respectively,

$$
R_{\mathrm{App}}^{\mathrm{MRT} 1}=\sum_{k=1}^{N_{u}} \log _{2}\left(1+\frac{P \beta_{k}^{2}\left\|\mathbf{F} \overline{\mathbf{h}}_{k}\right\|^{4}}{\sum_{i=1}^{N_{u}} \beta_{i}\left\|\mathbf{F} \overline{\mathbf{h}}_{i}\right\|^{2}}\right)
$$

and

$$
R_{\mathrm{App}}^{\mathrm{MRT} 2}=\sum_{k=1}^{N_{u}} \log _{2}\left(1+\frac{P \beta_{k}\left\|\mathbf{F} \overline{\mathbf{h}}_{k}\right\|^{2}}{N_{u}}\right) .
$$

Case 3: In the case that $K_{k}, K_{j} \rightarrow \infty$, if the users are located in the same direction, then (24) and (28) are reduced to, respectively,

$$
R_{\mathrm{App}}^{\mathrm{MRT} 1}=\sum_{k=1}^{N_{u}} \log _{2}\left(1+\frac{P \beta_{k}^{2}\left\|\mathbf{F} \overline{\mathbf{h}}_{k}\right\|^{2}}{\sum_{j \neq k} P \beta_{k} \beta_{j}\left\|\mathbf{F} \overline{\mathbf{h}}_{k}\right\|^{2}+\sum_{i=1}^{N_{u}} \beta_{i}}\right)
$$

and

$$
R_{\mathrm{App}}^{\mathrm{MRT} 2}=\sum_{k=1}^{N_{u}} \log _{2}\left(1+\frac{P \beta_{k}\left\|\mathbf{F} \overline{\mathbf{h}}_{k}\right\|^{2}}{P \beta_{k}\left(N_{u}-1\right)\left\|\mathbf{F} \overline{\mathbf{h}}_{k}\right\|^{2}+N_{u}}\right) .
$$

For either $K_{k}=0$ or $K_{k} \rightarrow \infty$ cases, if the short-term normalization is employed at the $\mathrm{BS}$, the user with lowest $\beta_{k}$ can obtain relatively higher received SINR when compared with the long-term normalization case. On the contrary, for long-term normalization, users with higher $\beta_{k}$ will be allocated with more power on their data streams. Thus, we can conclude that when MRT precoding is adopted, long-term normalization is preferred for the users who have stronger channel quality, while short-term normalization improves the quality of users in 
poor propagation condition. Similar to the MRC receiver, the conclusion about the effect of orthogonality condition among LoS paths from different users still holds for MRT precoders.

\section{Analog Beam Selection Schemes}

According to previous analysis, analog beamformer $\mathbf{F}$ directly determines the achievable rate of the hybrid beamforming system. Hence, it is crucial to choose proper beams that will be used for the design of phase shifter networks. As mentioned above, the design of $\mathbf{F}$ is transformed into the design of $\Psi$, which is exactly the selecting matrix of DFT beams $\mathbf{U}$. Here, we compare two designs of $\boldsymbol{\Psi}$.

\section{A. Exhausted Searching Scheme}

For the purpose of maximizing the achievable rate of this multiuser system, the first scheme is based on the previous analytical results. According to the applications including the direction of transmission link and the normalization method, we use the corresponding approximation expression to help choose DFT beams. It is an exhausted searching scheme which traverses all the possible beam combinations and jointly exploits the optimum beams for all the RF chains.

Assume that perfect CSI of all the users is known at the BS. As mentioned above, each of the $N_{s}$ RF chains will be assigned with a DFT beam selected from the $M$-sized codebook. One beam can be adopted by more than one RF chains. Therefore, there are totally $M^{N_{s}}$ beam combinations. Assume we adopt the MRC receiver in the uplink. Then after evaluating the achievable rate of each beam combination, we get the optimum combination that maximizes (9), i.e.,

$$
\left(i_{1}, \ldots, i_{N_{s}}\right)=\underset{i_{1}, \ldots, i_{N_{s}}=1, \ldots, M}{\arg \max } R_{\mathrm{App}}^{\mathrm{MRC}}
$$

This achievable rate based exhausted searching utilizes Theorems 1-3 and strives for the optimization of the rate performance. However, it is an incredibly time-consuming solution when $M$ or $N_{s}$ grows large.

\section{B. Per-User Selection Scheme}

To avoid the drawback of the proposed exhausted searching scheme, we further devise a per-user selection scheme which can greatly reduce the computation time.

This scheme needs the analog beamforming to concentrate on the major direction of all the serving users. For fairness, we strive to make each user utilize equal number of RF chains. Denote $C=\left\lfloor N_{s} / N_{u}\right\rfloor$, where $\lfloor\cdot\rfloor$ represents rounding a decimal to its nearest lower integer. Then, each of the first $N_{u}-1$ users is allocated with $C \mathrm{RF}$ chains, and the $N_{u}$ th user is allocated with $N_{s}-C\left(N_{u}-1\right)$ RF chains.

Assume that BS knows the covariance matrix of each user's channel, i.e.,

$$
\mathbf{R}_{k}=\frac{1}{T} \sum_{t=1}^{T} \mathbf{h}_{k}(t) \mathbf{h}_{k}^{H}(t)
$$

which can be singular value decomposed by

$$
\mathbf{R}_{k}=\mathbf{U}_{k}^{H} \mathbf{A} \mathbf{V}_{k}
$$

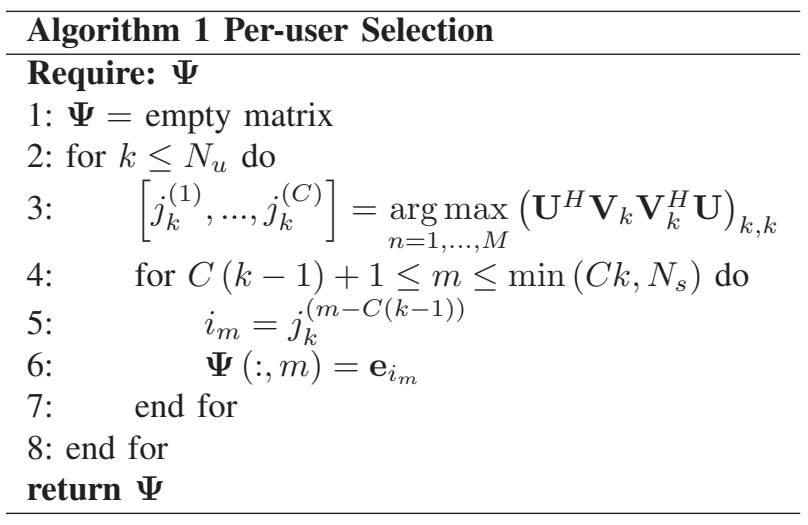

for $k=1, \ldots, N_{u}$. In order to cover the main lobes of all the user channels, we pick out $\mathbf{V}_{k} \in \mathbb{C}^{M \times M}$ to help us select beams for user $k$. The per-user beam selection scheme is conducted through Algorithm 1, where $\mathbf{A}_{m, n}$ denotes the $(m, n)$ th entry of matrix $\mathbf{A}$.

Obviously, each user does not have to evaluate the huge number of beam combinations. Instead, the only need is to calculate the propagation energy of $M$ beams. This per-user selection scheme reduces the computation time from $M^{N_{s}}$ to $M \times N_{u}$, which is more acceptable for practical use.

\section{NumericAl RESUlts}

To evaluate the achievable rate approximations and the proposed two kinds of beam selection schemes, we use simulations and display the numerical results here.

Fig. 2 gives the comparison between Monte Carlo and approximation results of the uplink rate. In this simulation, $M=512, N_{u}=4, S N R=10 \mathrm{~dB}$, which measures the average transmit power of each user against the noise on each BS antenna. Considering the running time, the per-user selection scheme is adopted instead of the exhausted searching scheme. We can find from the figure that the approximations approach their Monte Carlo results closely, which insures the reliability of the derived beam selection scheme. We know that the Ricean factor $K$ plays an important role in the uplink rate. It shows that when $K$ is small, which means the channel is almost Rayleigh distributed, the performance of the multiuser system is not good enough due to the severe interuser interference. When $K$ grows to infinity, the achievable rate converges to a fixed value, which verifies our previous discussions. Moreover, if we increase $N_{s}$, the performance of the hybrid beamforming system is improved significantly.

Fig. 3 shows the performance of the approximation results of the downlink rate. The configuration is same with Fig. 2 except that the downlink SNR describes the total transmit power at the BS side against the noise on each user antenna which is set to $10 \mathrm{~dB}$. Similarly, the increase of either $N_{s}$ or $K$ is preferable for MRT precoders and the downlink achievable rate converges when $K$ grows towards infinity. We can also find from Fig. 3 that the achievable rate performance of long-term normalization and short-term normalization is unpredictable since they have different advantages. 


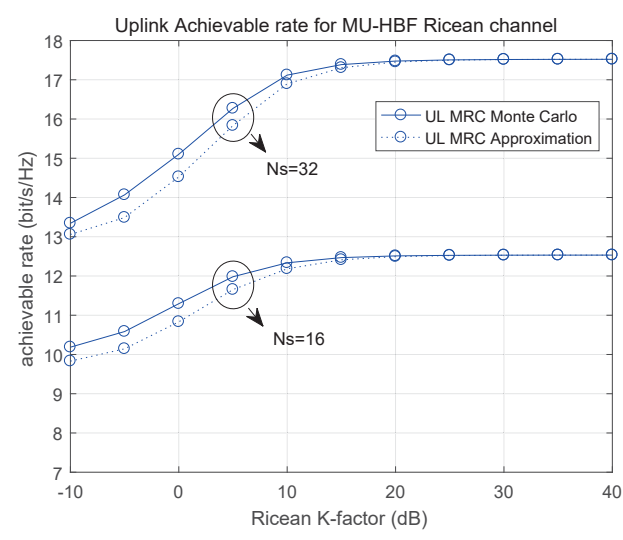

Fig. 2. Uplink achievable rate versus $K$ for hybrid BF multiuser system when $N_{s}=16$ and 32 .

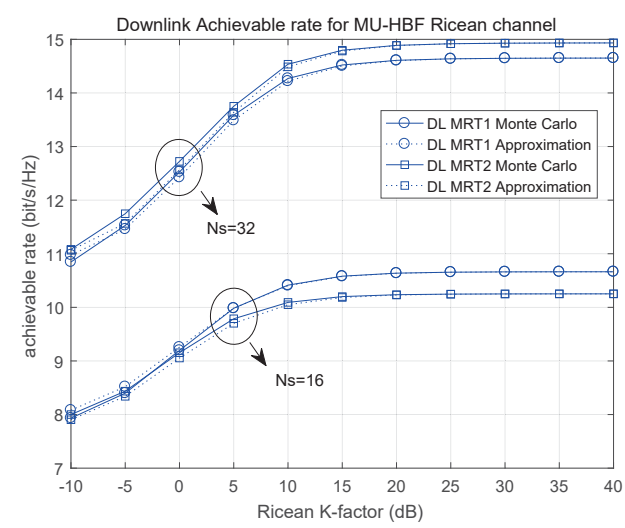

Fig. 3. Downlink achievable rate versus $K$ for hybrid BF multiuser system when $N_{s}=16$ and 32 .

Besides the rate analysis, we further derive two kinds of analog beam selection schemes. Fig. 4 compares the performance of the exhausted searching scheme with that of the per-user selection scheme in the condition that $M=30, N_{s}=$ $4, N_{u}=2$ and MRC receiver is adopted in the uplink. It can be seen that the performance of the per-user selection scheme is closely approaching to that of the exhausted searching scheme, but the amount of computation time unit is reduced from $30^{4}$ to $30 \times 2$. If we further increase $M$ or $N_{s}$, the exhausted searching scheme will be incredibly time-consuming.

\section{CONCLUSION}

This paper studied the beam selection schemes of the DFTbased multiuser hybrid beamforming system in TDD mode and Ricean fading scenario. With CSI, the uplink rate approximation of MRC receiver and the downlink rate approximations of MRT precoder were formulated. Analytical and numerical results showed that the orthogonality condition among LoS paths from different users directly determines the performance of MRC receiver and MRT precoder. Based on the analytical results, an exhausted searching scheme and a low-cost per-user selection scheme were proposed to select the DFT beams.

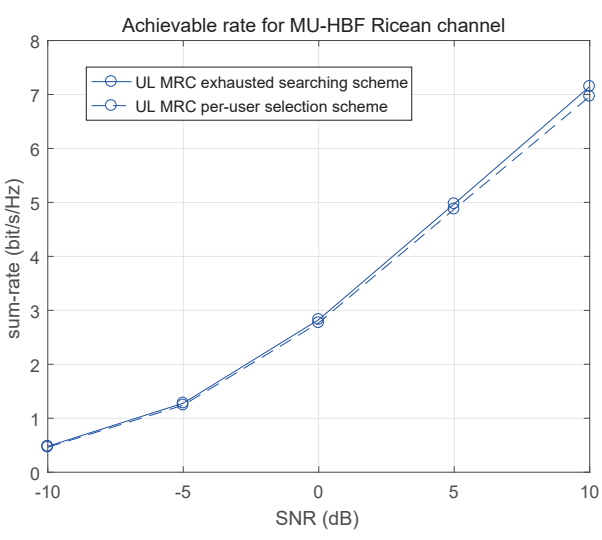

Fig. 4. Comparison of the exhausted searching scheme and the per-user selection scheme when MRC receiver is adopted in the uplink.

\section{ACKNOWLEDGMENT}

This work was supported in part by the National Science Foundation of China (NSFC) for Distinguished Young Scholars of China with Grant 61625106, the National Natural Science Foundation of China under Grant 61531011, and the Hong Kong, Macao and Taiwan Science and Technology Cooperation Program of China (2016YFE0123100). This work was also supported in part by the National Natural Science Foundation of China (NSFC) under Grants 61671251 and the Natural Science Foundation Program through Jiangsu Province of China under Grant BK20150852.

\section{REFERENCES}

[1] E. Larsson, O. Edfors, F. Tufvesson, and T. L. Marzetta, "Massive MIMO for next generation wireless systems," IEEE Commun. Mag. vol. 52, no. 2, pp. 186-195, Feb. 2014.

[2] J. G. Andrews, S. Buzzi, W. Choi, S. V. Hanly, A. Lozano, A. C K. Soong, and J. C. Zhang, "What will 5G be?" IEEE J. Sel. Areas Commun., vol. 32, no. 6, pp. 1065-1082, Jun. 2014.

[3] J. Hoydis, S. T. Brink, and M. Debbah, "Massive MIMO in the UL/DL of cellular networks: How many antennas do we need?" IEEE J. Sel. Areas Commun., vol. 31, no. 2, pp. 160-171, Feb. 2013.

[4] Y. Han, H. C. Zhang, S. Jin, X. Li, R. Yu and Y. Zhang, "Investigation of transmission schemes for millimeter-wave massive MU-MIMO systems," IEEE Syst. J., vol. 11, no. 1, pp. 72-83, Mar. 2017.

[5] A. Alkhateeb, O. El Ayach, G. Leus, and R. W. Heath, "Hybrid precoding for millimeter wave cellular systems with partial channel knowledge," in Proc. Inf. Theory Appl. Workshop, Feb. 2013, pp. 1-5.

[6] S. Han, C.-L. I, Z. Xu, and C. Rowell, "Large-scale antenna systems with hybrid analog and digital beamforming for millimeter wave $5 \mathrm{G}$," IEEE Commun. Mag., vol. 53, no. 1, pp. 186-194, Jan. 2015.

[7] D. A. Gore, R. W. Heath, and A. J. Paulraj, "Transmit selection in spatial multiplexing systems," IEEE Commun. Lett., vol. 6, no. 11, pp. 491-493, Nov. 2002.

[8] M. Matthaiou, C. Zhong, and T. Ratnarajah, "Novel generic bounds on the sum rate of MIMO ZF receivers," IEEE Trans. Signal Process., vol. 59, no. 9, pp. 4341-4353, Sept. 2011.

[9] Y. G. Lim, C. B. Chae, and G. Caire, "Performance analysis of massive MIMO for cell-boundary users," IEEE Trans. Wireless Commun., vol. 14, no. 12, pp. 6827-6842, Dec. 2015.

[10] L. Liang, W. Xu, and X. Dong, "Low-complexity hybrid precoding in massive multiuser MIMO systems," IEEE Wireless Commun. Lett., vol. 3, no. 6, pp. 653-656, Dec. 2014.

[11] Q. Zhang, S. Jin, K. K. Wong, H. Zhu, and M. Matthaiou, "Power scaling of uplink massive MIMO systems with arbitrary-rank channel means," IEEE J. Sel. Topics Signal Process., vol. 8, no. 5, pp. 966-981, Oct. 2014. 